\title{
THE FEATURES OF THE GEODETIC REFERENCE OF SATELLITE IMAGES
}

\author{
M.H. Gojamanov \\ Baku State University, Faculty of Geography, Dept. of Geodesy and Cartography \\ 23, Z.Khalilov Str., Baku, Azerbaijan, AZ-1148 \\ mgodja@yandex.ru \\ Commission I, ICWG I/V
}

KEY WORDS: Geodesy, Image, Resolution, Scale, Digital, Orthophototransformation, Spatial Georeferencing

\begin{abstract}
:
Space information is increasingly the main source of spatial data for many projects. Modern methods of processing of satelliif images and remote sensing data can get maps, showing the distribution of various objects and phenomena on the earth's surface. An important feature of future space vehicles is the high precision gridded images. And it allows creating maps of scale 1: 10,000 without using ground control points. As usual orthophotos and vector plans a large scale in the Countries of Independent States, created in the local area (local) coordinate system based on the coordinates in the coordinate system of 1942 (SF-42) to Gauss project. However, the spatial position of the satellite images is given in the WGS-84. Therefore there is a need to translate the raw materials of the WGS-84 in a local coordinate system of the project, in other words, the geodesic linking satellite images. Keys are needed for this conversion of coordinates from the local to the state or directly to the WGS-84. To determine the key of the transition there are used the different methods.

This article describes the features of geodetic reference satellite images with the use of software and hardware for "DPS-4.0 Talca." This software has the task of linking images with rational polynomial coefficients in the local coordinate system in the unknown parameters of translation to the coordinate system WGS-84. To do this, "DPS-Talca 4.0" has a special tool " linking images RPC in the local coordinate system."
\end{abstract}

\section{INTRODUCTION}

High demand on Earth remote sensing data (DRS) and satellite images caused the rapid development of computer technology and the improvement of geo-information technologies. Space data more frequently is the main of spatial data for many projects. With increasing resolution of images, increasing the geometric accuracy of the binding and the appearance of composite ortomozaik data DRS from the space begin to play the role of infrastructure, encompassing traditional maps.

Modern methods of processing of satellite images and remote sensing data, such as automatic classification, lineament analysis, 3D modeling, produce maps, showing the distribution of various objects and phenomena on the earth's surface. These materials can be used for further spatial analysis and cartographic content geoportals, which, in turn, are an essential part of industry information systems and a source of information for management decision making.

\section{MAIN BODY OF TEXT}

Since 1999 globally developed and launched 11 civilian spacecraft (SC) with optical and electronic equipment, providing production of images with a resolution 0.4-1.0 m, which are widely used in land use, land use planning in conducting, planning and construction of engineering structures , the exploration of hydrocarbon fields, the creation of topographic maps and plans.

An important feature of future spacecraft is a high precision gridded image, which is achieved through the use of space platform with high stability and improved accuracy of determining the orientation of the satellite. Thus, the coordinate accuracy of the satellite images of World View-1 without using ground control points within the image - at 2 $\mathrm{m}$, the control points on neighbor - 3 - $3.5 \mathrm{~m}$. This allows to create maps ot scale 1: 10000 without using ground control points.

Recently, for the construction of the large-scale orthophotoplans and the vector plans more frequently using digital satellite images or digital aerial photographs. As usual orthophotoplans and large -scale vector plans are created in the local area (local) coordinates in Gauss.

The local coordinate system in the Countries of Independent States, as a rule, based on the coordinates in the coordinate system of 1942 (SC-42) or 1995 (SC-95). On the other side the local coordinates system bear the imprint of all local deformations of State geodetic networks in the SC-42. At the same time, the spatial position of the images obtained from satellites, for example, IKONOS, GeoEye, QuickBird, WorldView, Spot, etc., is given in common terrestrial coordinate system WGS-84, or it can lead to this coordinate system, using as called rational polynomial coefficients (RPS). Therefore, there is a need to translate the raw materials of the WGS-84 to a local coordinate system of the project, in other words, to the geodesic linking of satellite images. Keys are needed for this conversion (converting) the coordinates from the local to the State coordinate system or directly to the WGS-84 system. However, the keys can not be known.

To determine the key transition are used different methods. In order to reduce the influence of local deformations of the SGN in SC-42 when determining the transition keys in the local coordinate systems, is proposed firstly transition to a less deformed system SC-95, and then to the WGS-84. 
If the current deformations in the local coordinate system are small, it can restrict the adjustment of transition keys from State to local coordinate system.

At the present time has developed various software and hardware for aerospace information visualization, in*tt;ch fully automated the process of internal and mutual orientation parameters. In addition, these soft wares are targets tor binding RPC images in the local coordinate system with unknown parameters to conversion system WGS-84. When the geodetic reference of images is fulfilling by using the control points whose coordinates are known in the local coordinate system of work area, a transformation of coordinates from the WGS-84 to the local is doing through specialty program. The parameters of the conversion of coordinates remain hidden from the operator. For example, to software, "DPS-Talca 4.0" for this purpose has a special tool "A binding of the RPC images to the local coordinate system." To launch a task it is necessary to select from the menu "Local (Scheduled) coordinate system." At the same time before starting a task it have to import value of this $\mathrm{RPC}$, corresponding of satellite images of this work area.

In calculating the elements of geodetic reference are used the points at least one of the selected images with coefficients RPC. Using a single ground control points on a single image is achieved less accurate reference. To obtain reliable results requires a minimum of three reference points. The presence of redundant control points (more than three) will allow a more accurate binding of the images. In the software, "DPSTalca" to convert all of satellite images of the project with coefficients RPC to a local (local) coordinate system of the work area is performed by the command "Calculate the binding".

\section{CONCLUSIONS}

In the process of geodetic reference of the images must be selected all the pictures in the list. If the elements of orientation in the form of RPC coefficients give large discrepancy on control points and their number is not less than three, then helpful to choose just one shot. Because, when you run the task of transforming the space images to local coordinate system, the transformation can be done with some rotation relative to the true grid. If the transformation is performed on one image, then this rotation will be reduced and insignificant. After this action it should be calculated aerial triangulation block with the coefficients of RPC for all images.

The above described procedure can create orthophotoplans based on satellite imagery with a given rational polynomial coefficients in the local coordinate system without using transition keys.

\section{REFERENCES}

Savinykh, V., Tsvetkov, V., 2001. GIS analysis of remote sensing data. Kartgeotsentr-Geodezizdat, Moscow, 228 p. Earth remote sensing data / / Spatial data, 1- Moskow-2010pp.45-52.

http:// www / sovzond.ru / 